\title{
Do prophylactic anticonvulsant drugs alter the pattern of seizures after craniotomy?
}

\author{
P M Foy, D W Chadwick, N Rajgopalan, A L Johnson, M D M Shaw
}

\begin{abstract}
A total of 276 patients with a high risk of developing postoperative seizures were randomised to treatment with carbamazepine or phenytoin for six or 24 months, or to no treatment. No significant differences were found (though the confidence limits were fairly wide) between the regimes in respect of the incidence of seizures or death. In a substantial proportion of the patients postoperative epilepsy remained a continuing disability. A high incidence of drug-related side effects was found in the treatment groups. Prophylactic anticonvulsants cannot therefore be recommended routinely following supratentorial craniotomy.
\end{abstract}

(f Neurol Neurosurg Psychiatry 1992;55:753-757)

Postoperative epilepsy remains a major problem following supratentorial neurosurgery for non traumatic conditions. ${ }^{1}$ A number of studies have attempted to determine whether prophylactic anticonvulsant therapy would alter the incidence ${ }^{2-4}$ or natural history ${ }^{5}$ of postoperative epilepsy and if so, whether this change would be maintained for the duration of the therapy alone or might prevent the development of chronic epilepsy.

Whilst this question is of importance for the practical management of patients following craniotomy for non-traumatic pathologies, there may be wider implications for the management of epilepsy. Reynolds ${ }^{6}$ has produced evidence from a variety of sources to suggest that early control and treatment of epilepsy may be important in determining the longterm outcome and in particular in preventing the development of chronic epilepsy. Postcraniotomy seizures may test this hypothesis because neurosurgical practice has used anticonvulsant prophylaxis in patients with a high risk of developing seizures.

A randomised prospective pragmatic clinical trial of prophylactic treatment policies has been undertaken, including a control group of untreated patients.

\section{Methods \\ a) Patients}

In previous studies ${ }^{15}$ high risk conditions, with an incidence of postoperative seizures of at least $20 \%$, have been identified. These include anterior communicating and middle cerebral artery aneurysms, arteriovenous malformations (AVMs), spontaneous intracerebral haematoma, meningioma and abscess. Other authors ${ }^{7}$ have reported that the subfrontal approach to pituitary masses also carries a high risk. Such patients, together with those having other suprasellar midline benign pathology, for example, craniopharyngioma have been included in this study (table 1). Any patient with a previous history of seizures was excluded as were those under the age of eighteen,

Table 1 Characteristics of randomised patients

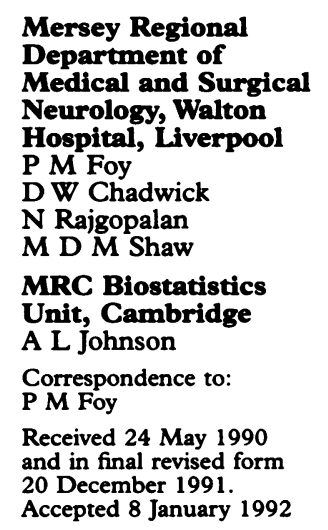

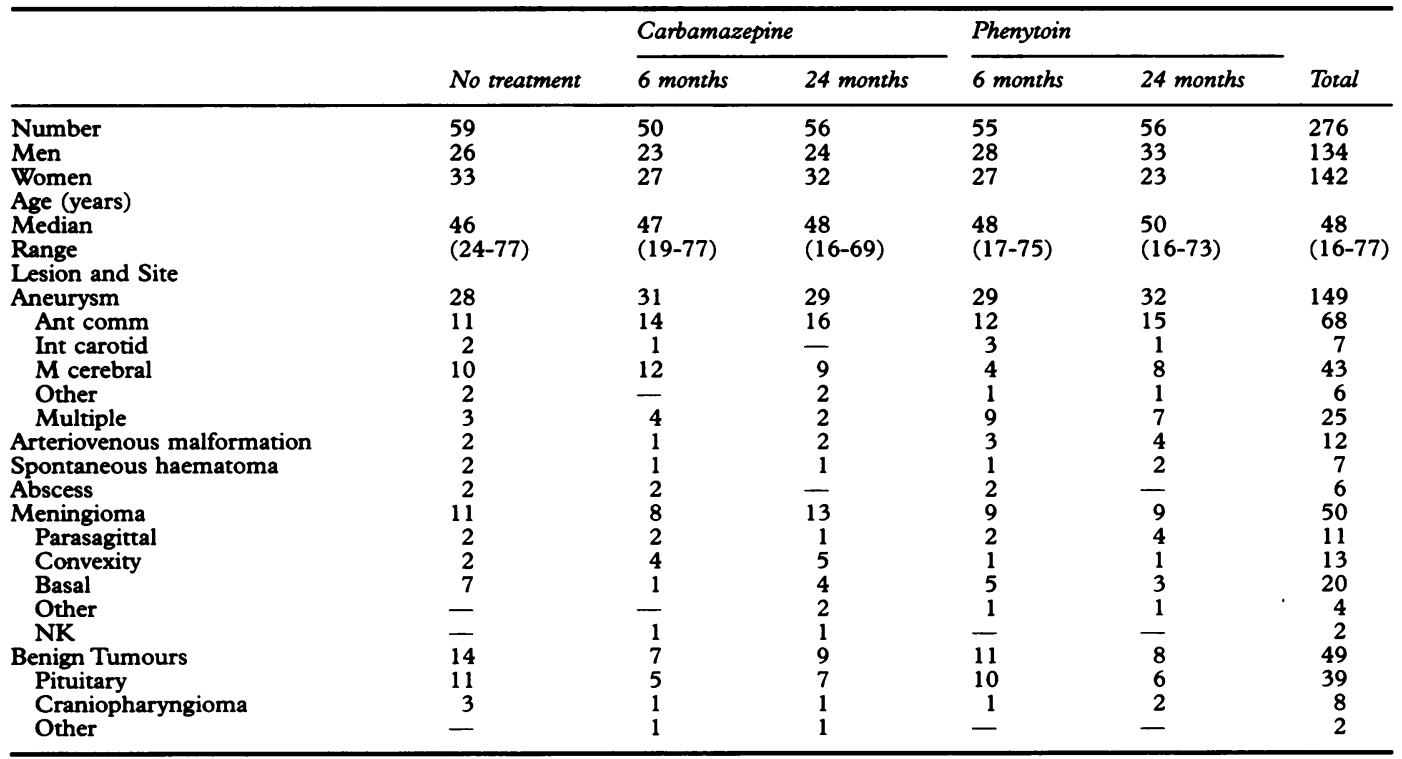


pregnant women and those with chronic renal, liver or cardiac disease. Table 2 summarises the details of the operative procedures. Manipulation of the brain surface refers to dissection of the lesion off the cortical surface of the brain, for example, as in the removal of a convexity meninglioma.

\section{b) Study design}

A total of 276 consecutive patients from the high risk groups who were admitted to the Mersey Regional Department between January 1981 and January 1986, and undergoing supratentorial craniotomy were randomised before surgery to no treatment or to treatment with carbamazepine or phenytoin. Patients allocated antiepileptic drugs were randomised equally to six months or two years treatment. Patients were stratified for pathological groups, for example, aneurysm, meningioma, before randomisation which used a system of blocks (size of 5) from prepared lists to ensure balance within groups. The first 102 patients were randomised to treatment with carbamazepine or phenytoin for six months or 24 months. Since analysis showed little difference in the incidence of postoperative seizures in this group relative to a retrospective study of postoperative seizures, ${ }^{2}$ the subsequent 176 patients were randomised equally between policies of no treatment, treatment with carbamazepine and treatment with phenytoin.

Assuming an average of three years of follow up with 607 seizure-free in the treatment groups and a randomisation ratio of $4: 1$ (treated:untreated), recruitment of 240 patients gives $80 \%$ power to detect a difference of $20 \%$ in seizure free rates using the log rank statistic at $p=0.05$. With 240 patients randomised equally to the two drugs or the two durations of treatment, there is $\mathbf{8 0 \%}$ power to detect comparable differences. The 276 patients included in the study therefore provide reasonable power to detect substantive differences between the different regimes; the power to detect intermediate but clinically important effects (10-15 differences in seizure-free rates) is poor.
Patients allocated to carbamazepine were loaded with $200 \mathrm{mg} 6$ hourly during the 24 hour period preoperatively and $200 \mathrm{mg} 8$ hourly thereafter. Phenytoin was administered intravenously in a dose of $15 \mathrm{mg} / \mathrm{kgm}$ for at least 24 hours before surgery and thereafter $100 \mathrm{mg} 8$ hourly orally or intravenously. Myocardial ischaemia, bradycardia or a conduction block were contra-indications to loading intravenously and such patients were given phenytoin orally.

Serum anticonvulsant levels were estimated postperatively in many patients, and at one week, three, six and nine months, one year and two years while the patients were on active therapy.

Follow up in survivors was for a minimum of three years and a maximum of eight years. The occurrence of a seizure or death was counted as the end point of this part of the study and antiepileptic drug regime altered as clinically indicated. Observation of the patient after seizures was continued to determine the natural history of the seizure disorder. Intervals from operation to occurrence of the first seizure, death or the last date of follow up, whichever was the earlier, were calculated. Patients who died after randomisation but preoperatively or those who died or had a seizure within a week of operation were assigned a follow up interval of one week. Kaplan-Meier plots were used to display actuarial seizure-free curves and the log rank statistic was used for comparisons between groups. Prognostic factors influencing outcome have been assessed using the Cox proportional hazards regression model.

\section{Results}

Of the 276 patients entered into this study, 103 $(37 \%)$ suffered at least 1 seizure. Twenty $(20 \%)$ of these patients and a further 44 patients who were seizure-free died during the study period. A total of 129 patients were alive and seizure-free at the last follow up. One hundred and fourteen were followed up for at least three years, 10 for between two and three

Table 2 Characteristics of operation

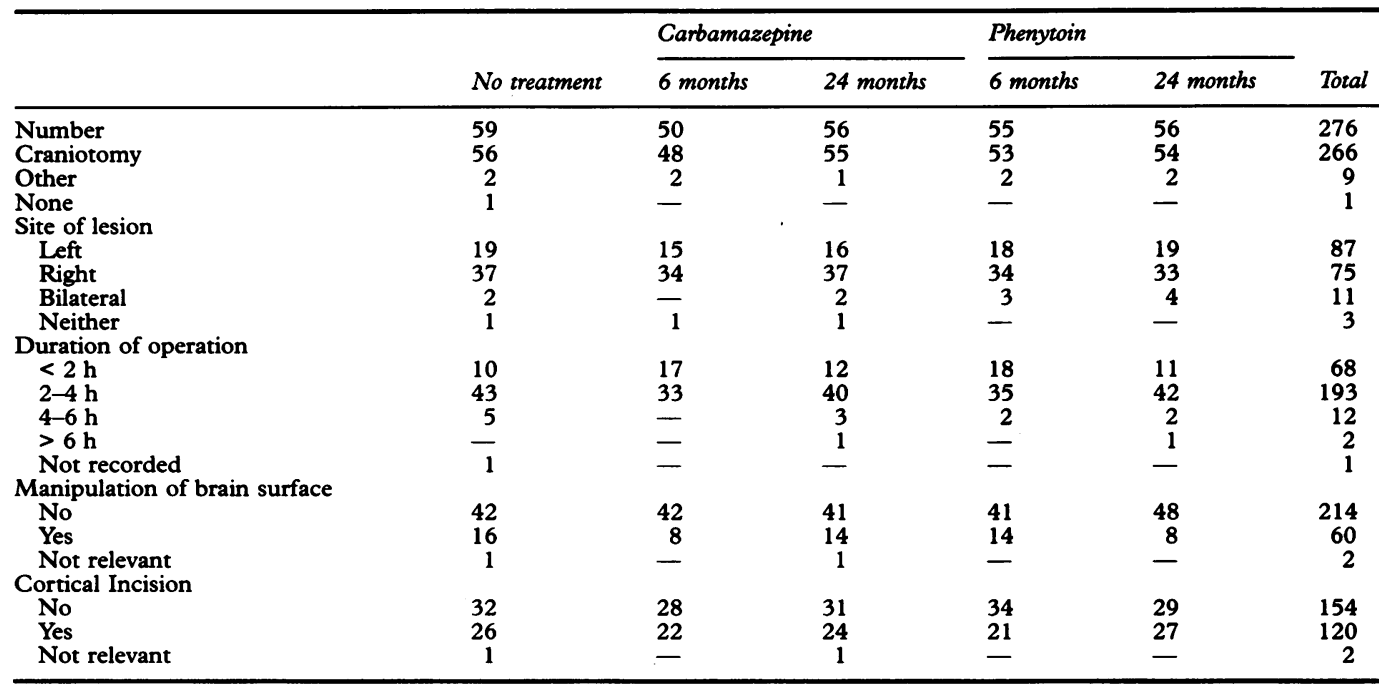


years. Only 5 were lost to follow up within two years of randomisation. The median follow up interval (all patients) was four years. Table 3 shows the actuarial percentages free of seizures up to four years of follow up. By one year $42 \%$ of all patients had had at least one seizure or died $(95 \%$ CL: 37,48$)$; by four years $53 \%$ (95\% CL: 47,59).

Figure 1 shows actuarial percentages seizure-free for no treatment, six months and two years treatment. There is no significant effect of treatment $\left(x^{2}=2 \cdot 2, \mathrm{df}=2, \mathrm{p}=0 \cdot 33\right)$ though the confidence intervals are quite wide and there is no trend for the two years of treatment to be more effective than either no treatment or six months treatment (table 3 ). It is clear, however, that some benefit of two years of treatment is apparent by six months, even though at this stage, treatment in this group does not differ from the group randomised to six months of therapy. There appears to be little to choose between the two drugs though the confidence intervals do not preclude differences as large as $20 \%$ by one year (table 3).

A number of factors appear to influence the occurrence of seizures including pathology. Figure 2 illustrates the actuarial percentages free from seizures in the largest groups of patients (pituitary tumours, aneurysms and meningiomas $)\left(x^{2}=16 \cdot 8, d f=2, p=0 \cdot 0002\right)$. Other factors influencing outcome were duration of operation $\left(<2,2-4,>4\right.$ hours) $\left(x^{2}=\right.$ $8 \cdot 2, \mathrm{df}=2, \mathrm{p}=0.016)$ and manipulation of the surface of the brain during surgery, that is, dissection of the lesion from the cortical surface $\left(x^{2}=7 \cdot 2, \mathrm{df}=1, \mathrm{p}=0.007\right)$. Left sided or bilateral lesions appeared to carry a higher risk of seizures than right sided lesions $\left(x^{2}=13 \cdot 1, \mathrm{df}=2, \mathrm{p}=0.0001\right)$. Site of operation, sex of patient and cortical incision during surgery did not appear to influence the incidence of seizures: nor did the Hunt and Hess grades in patients with subarachnoid haemorrhage.

Cox proportional hazards regression model was used to adjust the treatment effect for all the above factors except the Hunt and Hess score; the overall treatment effect was still not significant $(\mathrm{LRATIO}=5 \cdot 5, \mathrm{df}=4, \mathrm{p}=0 \cdot 24)$.
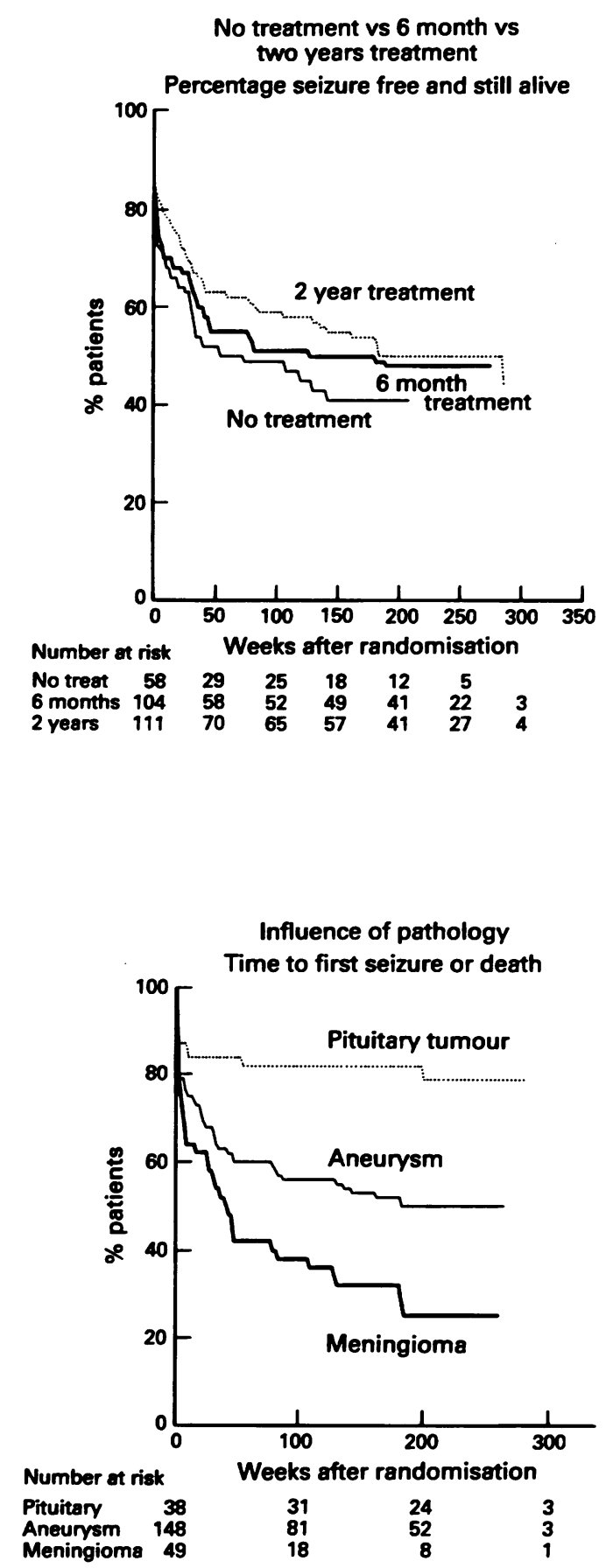

Table 3 Patient status at last follow up and actuarial \% seizure free by treatment group

\begin{tabular}{|c|c|c|c|c|c|c|c|c|c|}
\hline \multirow[b]{3}{*}{ Treatment group } & \multirow{3}{*}{$\begin{array}{l}\text { Number } \\
\text { patients }\end{array}$} & \multicolumn{4}{|c|}{ Status at last follow up } & & & & \\
\hline & & \multicolumn{2}{|c|}{ Seizure free } & \multicolumn{2}{|c|}{ Seizures } & \multicolumn{4}{|c|}{ Actuarial \% surviving seizure-free after selected intervals } \\
\hline & & Alive & Dead & Alive & Dead & & $1 y$ & $2 y$ & $4 y$ \\
\hline \multirow{4}{*}{$\begin{array}{l}\text { No treatment } \\
\text { Carbamazepine } \\
(6 \mathrm{~m}) \\
(24 \mathrm{~m}) \\
\text { Subtotal } \\
\text { Phenytoin } \\
(6 \mathrm{~m}) \\
(24 \mathrm{~m}) \\
\text { Subtotal } \\
\text { Allocated drugs } \\
\quad(6 \mathrm{~m}) \\
\quad(24 \mathrm{~m}) \\
\text { Subtotal } \\
\text { All patients } \\
\text { Differences between } \\
\text { group (95\% CL) }\end{array}$} & 59 & 25 & 9 & 21 & 4 & 63 & 52 & 49 & 41 \\
\hline & $\begin{array}{r}50 \\
56 \\
106\end{array}$ & $\begin{array}{l}22 \\
29 \\
51\end{array}$ & $\begin{array}{r}7 \\
7 \\
14\end{array}$ & $\begin{array}{l}19 \\
17 \\
36\end{array}$ & $\begin{array}{l}2 \\
3 \\
5\end{array}$ & $\begin{array}{l}74 \\
73 \\
74\end{array}$ & $\begin{array}{l}58 \\
68 \\
63\end{array}$ & $\begin{array}{l}50 \\
64 \\
58\end{array}$ & $\begin{array}{l}45 \\
52 \\
49\end{array}$ \\
\hline & $\begin{array}{r}55 \\
56 \\
111\end{array}$ & $\begin{array}{l}26 \\
27 \\
53\end{array}$ & $\begin{array}{r}8 \\
13 \\
21\end{array}$ & $\begin{array}{l}14 \\
12 \\
26\end{array}$ & $\begin{array}{r}7 \\
4 \\
11\end{array}$ & $\begin{array}{l}60 \\
70 \\
63\end{array}$ & $\begin{array}{l}53 \\
57 \\
55\end{array}$ & $\begin{array}{l}53 \\
53 \\
53\end{array}$ & $\begin{array}{l}51 \\
47 \\
49\end{array}$ \\
\hline & $\begin{array}{l}105 \\
112 \\
217 \\
276\end{array}$ & $\begin{array}{l}48 \\
56 \\
104 \\
129 \\
\text { Some } \\
\text { Carba } \\
24 \mathrm{~m}\end{array}$ & $\begin{array}{l}15 \\
20 \\
35 \\
44 \\
\text { none } \\
\text { lazepine } \\
6 \mathrm{~m}\end{array}$ & $\begin{array}{r}33 \\
29 \\
62 \\
83 \\
- \text { Pheny }\end{array}$ & $\begin{array}{r}9 \\
7 \\
16 \\
20 \\
\text { oin }\end{array}$ & $\begin{array}{r}67 \\
72 \\
68 \\
67 \\
6(-8,20) \\
11(-2,23) \\
6(-7,18)\end{array}$ & $\begin{array}{l}55 \\
63 \\
59 \\
58 \\
7(-8,21) \\
8(-5,21) \\
7(-6,20)\end{array}$ & $\begin{array}{l}51 \\
59 \\
55 \\
54 \\
7(-8,21) \\
4(-9,18) \\
7(-6,21)\end{array}$ & $\begin{array}{l}48 \\
49 \\
49 \\
47 \\
8(-6,23) \\
0-(14,13) \\
1(-12,15)\end{array}$ \\
\hline
\end{tabular}


However, this multivariate model suggested a linear trend in treatment effect from no treatment to six months treatment to 24 months treatment with coding $0,1,2$ respectively $\left(\mathrm{x}^{2}=\right.$ $4 \cdot 7, \mathrm{df}=1, \mathrm{p}=0.031$; odds ratio $(95 \% \mathrm{CL})$ : $0.75(0.58,0.98)$.

Of those who developed status epilepticus in the first week $8 \%$ were treated with carbamazepine, $5 \%$ with phenytoin and $2 \%$ had no treatment. However, the numbers are too small for detailed analysis to discover if there is any treatment effect.

A total of 194 patients (89\%) claimed compliance with the treatment policy and $23 \%$ of these had seizures within the treatment period (six or 24 months). Twenty three patients $(11 \%)$ were non-compliant either because they discontinued treatment on their own initiative or because it was stopped by their physicians. Thirty per cent of such patients suffered seizures. This difference is not statistically significant (odds ratio $=1 \cdot 31 ; 95 \%$ CL, $0.53,3.25$ ), but does not exclude important effects for non compliance.

Serum anticonvulsant levels were not monitored in all patients (table 4), and in some patients in whom they were monitored the levels were never in the optimal range, particularly if patients had been randomised to phenytoin. However patients whose anticonvulsant levels were not monitored appeared no more likely to have seizures than those whose levels were (odds ratio $=0.77$; $95 \%$ CL, $0.39,1.53)$. Those patients who did not have an optimal serum level appeared no more likely to have seizures than those who did (odds ratio $=0.64 ; 95 \%$ CL $0.29,1.39$ ).

Early anticonvulsant treatment did not affect the longer-term response of epilepsy to anticonvulsant drugs. Only 39 of 102 patients with seizures had a single fit. Of 207 patients who survived and who were followed up to two or more years, 23 (12\%) had experienced seizures in the year preceding the date of the last followup. This included $4(17 \%)$ of the 23 patients with seizures randomised to no antiepileptic therapy and $19(14 \%)$ of 135 patients randomised to 1 of the 4 active treatments (odds ratio $=1.24,95 \% \mathrm{CL}, 0.39,3.96)$. Of those with more than one seizure and surviving at least two years, $37 \%$ experienced multiple seizures in the last year of follow up and $55 \%$ in the last two years.

We did not find that the occurrence of seizures within the first postoperative week (that is, early seizures) increased the likelihood of late epilepsy. Of 255 patients surviving more than one week 8 of 34 patients with early

Table 4 Blood levels and incidence of seizures

\begin{tabular}{llll}
\hline & Carbamazepine & Phenytoin & Total \\
\hline $\begin{array}{l}\text { No levels } \\
\text { Always }\end{array}$ & $29(12)$ & $24(9)$ & $53(21)$ \\
$\begin{array}{l}\text { Sub optimal } \\
\text { At least one }\end{array}$ & $2(1)$ & $50(13)$ & $52(14)$ \\
$\quad \begin{array}{l}\text { optimal } \\
\text { Total }\end{array}$ & $75(27)$ & $37(14)$ & $112(41)$ \\
& $106(40)$ & $111(36)$ & $217(76)$
\end{tabular}

Figures in brackets are number of patients in each group with seizures during follow up. seizures developed late epilepsy compared with 63 of 221 who did not have early seizures (odds ratio $=0.83 ; 95 \% \mathrm{CL}, 0.36,1 \cdot 87)$.

Side effects were relatively common in antiepileptic drug treated patients. The most common side effect necessitating a change or cessation in therapy was acute allergic skin rash. Rashes occurred in 28 and other drugrelated side-effects in 3 of the 217 patients who were treated with antiepileptic drugs. Of these 31 patients, 8 or $25 \%$ had seizures which is a lower rate than other groups in the study. A further 6 patients developed signs of drug intoxication.

\section{Discussion}

This study confirms that there is a high incidence of postoperative seizures and epilepsy after supratentorial craniotomy for certain nonprogressive pathologies. Previous studies $^{15}$ have indicated that the incidence of seizures varies with pathology, being less than $8 \%$ in patients with internal carotid artery aneurysms and $38 \%$ in patients with middle cerebral artery aneurysms. The presence of haematoma increases the risk in patients with aneurysms to $42 \%$. The overall incidence of seizure or death in this prospective study of high risk patients was $53 \%$ by four years (hazard rate $(95 \% \mathrm{CL}): 0 \cdot 19(0 \cdot 16,0.22)$ per year) compared with a risk of $34 \%$ by five years for similar pathologies in a previous retrospective study. ${ }^{15}$ The difference may indicate more complete follow up in a prospective study.

North ${ }^{4}$ advocated prophylactic antiepileptic drugs in patients whose risk of developing postoperative seizures is in excess of $15 \%$. However, a prospective study of 102 patients given prophylactic anticonvulsant drugs did not suggest an important reduction in the incidence of seizures when compared with historical controls. ${ }^{2}$ We therefore included a no treatment control group in this prospective study.

There was a trend for antiepileptic drugs to reduce the incidence of postoperative seizures and epilepsy during the time that they were taken. This reduction was small being on average less than $10 \%$ but the confidence limits are such that a reduction of $20 \%$ (table 3 ) could not be ruled out. We found minimal differences between no treatment and treatment with phenytoin or carbamazepine for six months. Patients treated with these drugs for two years appeared to have a somewhat better outcome. Surprisingly some difference was apparent in this group by six months despite the treatment policy not differing from that experienced by patients randomised to treatment for six months. At four years the difference between the two treatment policies is small $(1 \%)$ though again the confidence limits are too wide to exclude a $15 \%$ difference. To reduce the confidence limits, a larger multicentre trial in high risk patients would be needed. North et $a^{3}{ }^{3}$ suggested that phenytoin when given prophylactically was effective in reducing the incidence of seizures between 7 
and 70 days postoperatively. Our study fails to confirm this finding. A 5\% incidence of seizures was found to occur in each of the groups between the second and thirteenth postoperative weeks inclusive. Seizures and status epilepticus were not reduced within the first week postoperatively by either anticonvulsant drug.

A number of factors appeared to affect the incidence of seizures/death. The pathology was of major importance with the highest risk in patients with meningiomas $(75 \%$ by four years), an intermediate risk for anterior and middle cerebral aneurysms $(51 \%$ by four years) and the lowest risk for pituitary tumours ( $21 \%$ by four years). Operative factors also influenced the risk of seizures. Longer operations and those associated with dissection of the lesion away from the surface of the brain were most likely to be complicated by seizures. It appeared that left sided lesions and operations were more likely to be complicated by epilepsy.

The small and uncertain effect of antiepileptic drug treatment in this study might be related to non-compliance and failure to achieve optimal blood levels. Some patients admitted to non-compliance for a variety of reasons. These patients were no more likely to have seizures than those who claimed to be compliant. We did not achieve our objective of detailed blood level monitoring throughout the antiepileptic drug treatment. Of those blood levels available a significant proportion of phenytoin levels were suboptimal and it could be argued that more rigorous monitoring might improve results. However, satisfactory carbamazepine levels were achieved whenever these were monitored. As there was little difference between the outcome for phenytoin or carbamazepine it is doubtful that more rigorous blood level monitoring would have achieved better results unless phenytoin has a greater prophylactic action than carbamazepine, which seems unlikely. This study was pragmatic in its objectives and the compliance and ability to obtain blood level monitoring reflects what is likely to be achieved in routine clinical practice in a group of patients who have undergone craniotomy and had not previously suffered seizures.

The study can be criticised becaused the no treatment group was recruited only in the latter part of the study. However, we have no evidence that characteristics of patients recruited in the 2 phases of the study differed, or that the incidence of seizures or death altered in randomised treatment groups during the course of the study. It is doubtful that this influenced our results. Any treatment effects due to anticonvulsant drugs in this population of patients were achieved at the cost of side effects, particularly allergic skin rashes. ${ }^{8}$ This study provides little evidence to support the routine use of prophylactic antiepileptic drugs and there is no evidence that prophylactic treatment made epilepsy easier to control with antiepileptic drugs once it developed. The proportion of patients continuing to have seizures during the last year or two years of follow up was not influenced by whether they had taken early treatment. Such seizures continued to be a problem for a high percentage of the patients who have any seizures following craniotomy. In this study the occurrence of seizures and epilepsy is determined by the pathology and the nature of the preoperative brain injury and not to a major degree by effects of antiepileptic drugs on the process of epileptogenesis. These results are similar to those reported by Temkin et $a l^{9}$ in a large randomised study of phenytoin head injured patients.

We cannot therefore recommend prophylactic anticonvulsant drugs after supratentorial craniotomy and would recommend that treatment should be delayed, at least until the occurrence of the first seizure after surgery.

1 Foy PM, Copeland GP, Shaw MDM. The incidence of post operative seizures. Acta Neurochir 1981;55:253-64.

2 Shaw MDM, Foy PM, Chadwick DW. The effectiveness of prophylactic anticonvulsants following neurosurgery. Acta Neurochir 1983;69: 253-8.

3 North JB, Penhall RK, Hanieh A, Frewin DB, Taylor WB. Phenytoin and postoperative epilepsy. $\mathcal{f}$ Neurosurgery 1983;58:672-7.

4 North JB. Anticonvulsant prophylaxis in neurosurgery, (Editorial). B f Neurosurg 1983;3:425-8.

5 Foy PM, Copeland GP, Shaw MDM. The natural history of postoperative seizures. Acta Neurochir 1981;57:15-22.

6 Reynolds EH. Early treatment and prognosis of epilepsy. Epilepsia 1983;28:97-106.

7 Cast IP, Wilson PJE. Pituitary tumours. A ten year survey. $\mathfrak{f}$ Neurol Neurosurg Psychiatry 1981;44:371.

8 Chadwick D, Shaw MDM, Foy P, Rawlins MD, Turnbull DM. Serum anticonvulsant concentrations and the risk of drug induced skin eruptions. $\Im$ Neurol Neurosurg Psychiatry 1984;47:642-4.

9 Temkin NR, Dikmen SS, Wilensky AJ, Reihm J, Chabal S, Winn HR.A randomized, double-blind study of phenytoin for the prevention of posttraumatic seizures. $N$ Engl $f$ Med 1990;323:497-502. 